\title{
Von der Kunstlehre des Verstehens zur radikalen Interpretation
}

\section{Hermeneutik und „theory of interpretation“}

Zwischen der kontinentalen Hermeneutik und dem, was im englischen Sprachraum „theory of interpretation“ heißt, gibt es nach wie vor einen Rezeptionsgraben. Warum bis heute so wenig daran gearbeitet wird, ihn zuzuschütten, ist nicht leicht zu erklären. Dass der Graben auf einen unauflöslichen Methodenkonflikt zwischen „kontinentaler“ und „analytischer“ Philosophie zurückgehe, ist eine bequeme Erklärung, die aber in der Sache nicht viel für sich hat. Der Hauptgrund dürfte der kontingente Umstand sein, dass es nur wenige Verstehenstheoretiker gibt, die sich in beiden Theorietraditionen gleichermaßen auskennen. Wer über Schleiermacher, Dilthey oder Gadamer arbeitet, ist in der Regel mit den Werken von Quines und Davidsons kaum vertraut - und vice versa. Hinzu kommt selbstverschuldete Unempfänglichkeit. Die kontinentale Hermeneutiktradition hat den Anschluss an die rasante Entwicklung der sprachphilosophischen Bedeutungstheorie in der zweiten Hälfte des 20. Jahrhunderts nicht ernsthaft gesucht. Umgekehrt konnte es noch vor wenigen Jahren geschehen, dass man, wenn man unter anglophonen analytischen Philosophen den Ausdruck ,"hermeneutics“ fallen ließ, die Rückfrage provozierte: „Herman who?“‘

Mein Beitrag kontrastiert Schleiermachers Kunstlehre des Verstehens mit einem Theoriestrang der Sprachphilosophie im 20. Jahrhundert, den man unter das Stichwort „Radikalisierung des Verstehensproblems“ stellen kann. Gemeint sind die Theorien der „radikalen Übersetzung“ und der „radikalen Interpretation“ von Willard Van Orman Quine (1908-2000) und Donald Davidson (1917-2003).

Eine direkte Verbindung zwischen Quine und der kontinentalen Hermeneutiktradition gibt es nicht. Es käme auch niemand auf die Idee, Quine als einen hermeneutischen Philosophen zu bezeichnen, wiewohl seine Theorie der radikalen Übersetzung sich mit einem hermeneutikverdächtigen Thema befasst, nämlich der Interpretation der Äußerungen eines Sprechers einer unbekannten Sprache unter stark erschwerten Bedingungen. Etwas anders verhält es sich mit Davidson, der 1949 bei dem deutschen Gräzisten Werner Jaeger in Harvard über Platons Dialog Philebos promoviert hat. Dieses Thema hatte er mit Gadamers Habilitationsschrift gemeinsam. Später sollte deutlich werden, dass Davidson und Gadamer noch mehr gemeinsam haben: Das Prinzip der wohlwollenden Interpretation (,principle of charity“), das in Davidsons Interpretationstheorie eine 
zentrale Rolle spielt, kann als das analytische Pendant zu Gadamers „Vorgriff auf Vollkommenheit“ gelten. Beide sind Nachfahren der Interpretationsmaxime, die in Georg Friedrich Meiers Versuch einer allgemeinen Auslegungskunst aus dem Jahre 1757 das Prinzip der „hermeneutischen Billigkeit“ heißt.

Davidsons Interpretationstheorie ist treffend als „eine Hermeneutik intelligenten Verhaltens“ bezeichnet worden, die „das Verstehen sprachlicher Äußerungen und die Erklärung nonverbalen Handelns zu einem einzigen Projekt integriert“. ${ }^{1}$ Sowohl die Interpretation von Äußerungen als auch die von Handlungen geschehen durch Zuschreibungen von Überzeugungen, Wünschen und Absichten. Wir interpretieren das sprachliche und nichtsprachliche Verhalten anderer Personen, indem wir es als Ausdruck von mentalen Einstellungen ansehen, die wir der Person im Zuge der Interpretation zuschreiben. Beim Unternehmen der radikalen Interpretation kennt der Interpret vorab keinerlei mentale Einstellungen des Sprechers, vielmehr müssen die Bedeutungen und die mentalen Einstellungen simultan und in Abhängigkeit voneinander ermittelt werden. Es gibt einen engen Zusammenhang zwischen dem, was jemand mit einer Äußerung meint, und dem, was er glaubt bzw. für wahr hält, aber der radikale Interpret ist mit dem methodischen Problem konfrontiert, dass keines der beiden Phänomene als unabhängiger Beleg für das andere fungieren kann. ${ }^{2}$ Man versteht eine (assertorische) Äußerung nicht, wenn man nicht weiß, welche Überzeugungen damit ausgedrückt werden, aber die Sprecherüberzeugungen kann der Interpret nicht identifizieren, solange er nicht weiß, was dessen Äußerungen bedeuten. Wenn dieser Zirkel sich nicht als hermeneutischer erweist, kann das Unternehmen nicht fortschreiten.

Davidson ist entsprechend der Auffassung, „daß die Interpretation der Intentionen, Überzeugungen und Worte eines Handelnden zu einem einzigen Vorhaben gehören, von dem man kein Teil für vollständig erachten kann, ehe der Rest beisammen ist. “3 Bezieht man nun noch den Umstand mit ein, dass auch Handlungen interpretationsbedürftig sind und ihrerseits, wenn erfolgreich interpretiert, Aufschluss über die mentalen Einstellungen des Akteurs verschaffen, so zeigt sich der wahre Umfang des hermeneutischen Zirkels: „Für Davidson sind [...] die Interpretation von Äußerungen und die Interpretation nonverbalen Handelns Teile ein und desselben Projekts: Der Radikalinterpret wird zum Hermeneuten allen intentionalen Handelns, sei es nun sprachlich oder nicht.“4

1 Kathrin Glüer, Donald Davidson zur Einführung, Hamburg 2003, 9.

2 Vgl. Donald Davidson, „Der Begriff des Glaubens und die Grundlage der Bedeutung“ (1974), in: ders., Wahrheit und Interpretation, Frankfurt am Main 1986, 204-223, hier 204- 208.

3 Donald Davidson, „Radikale Interpretation“ (1973), in: ders., Wahrheit und Interpretation, a.a.O. (Anm. 2), 183-203, hier 186.

4 Glüer, Davidson, a.a.O. (Anm. 1), 79 f. 
Gleichwohl hat sich für Davidsons Unternehmen der Ausdruck „theory of interpretation“ eingebürgert und nicht „Hermeneutik“. Der besagte Rezeptionsgraben ist einer der Gründe dafür. Es gibt in beiden Theorietraditionen Elemente, die für die andere Seite rote Tücher sind. So können viele analytische Philosophen mit der Rede von „Einfühlung“ wenig anfangen und halten die Idee des einfühlenden Nacherlebens fremder Gedanken für methodologisch naiv. Dass Schleiermacher das „divinatorische“ Moment der Auslegung mit den Vokabeln „ahn(d)en“ und „erraten“ charakterisiert, leistet diesem Bedenken noch Vorschub. ${ }^{5}$ Auf der anderen Seite erscheint Quines ausgeprägter Behaviorismus (den Davidson nicht teilt) aus Sicht der kontinentalen Hermeneutiktradition befremdlich. Gehört es nicht wesentlich zur hermeneutischen Kunst, im Zuge der Auslegung auf Gedanken, Gefühle, Einstellungen des Sprechers zu schließen? Quine schreibt mentale Einstellungen nur notgedrungen und stets nur so weit $\mathrm{zu}$, wie zur Erklärung des Verhaltens der Person unbedingt erforderlich. Zudem möchte er mentale Zustände nur als vorläufige Stellvertreter für dereinst zu entdeckende physische Korrelate verstanden wissen. ${ }^{6}$

\section{Radikalisierungen des Verstehensproblems}

Schleiermacher hat die „Auslegungskunst“ als das umfassende Unternehmen charakterisiert, „sich in den Besitz aller Bedingungen des Verstehens zu setzen [...] Das Geschäft der Hermeneutik darf nicht erst da anfangen, wo das Verständniß unsicher wird, sondern vom ersten Anfang des Unternehmens an, eine Rede verstehn zu wollen“. ${ }^{7}$ Eine Kunstlehre des Verstehens müsse zudem davon ausgehen, dass sich nicht das Verstehen von selbst ergibt, wie die „kunstlose Praxis“ annehme, sondern vielmehr das Missverstehen. ${ }^{8}$ Schleiermacher lässt in diesen Bemerkungen keinen

5 Es ist kritisch bemerkt worden, dass Schleiermachers Ausführungen zum divinatorischen Verstehen nicht gut an die zahlreichen Vorläuferprojekte des 17. und 18. Jahrhunderts angebunden sind: „Hätte Schleiermacher die Entwicklung der allgemeinen Hermeneutik besser gekannt, hätte er seinen Begriff des divinatorischen Verstehens in fruchtbarer Weise mit der Präsumtionsmethodologie der Aufklärungshermeneutiken vergleichen können. Leider blieb diese Auseinandersetzung aus.“ Oliver Scholz, Verstehen und Rationalität. Untersuchungen zu den Grundlagen von Hermeneutik und Sprachphilosophie, Frankfurt am Main 1999, 73 f.

6 Vgl. W.V.O. Quine, „Mind and Verbal Dispositions“, in: Mind and Language, ed. Samuel Guttenplan, London 1975, 83-95.

7 Die allgemeine Hermeneutik (1809/10), KGA II/4, $73 \mathrm{f}$.

8 „Die kunstlose Praxis geht davon aus, daß sich das Verstehen von selbst ergiebt“, die Hermeneutik als Auslegungskunst hingegen davon, „daß sich das Mißverstehen von selbst ergiebt 
Zweifel daran, dass die Hermeneutik grundlegend anzusetzen und das Verstehen stets als problematisch zu behandeln hat. Gleichwohl handelt es sich bei den Ansätzen Quines und Davidsons um eine Radikalisierung der hermeneutischen Fragestellung. Es lassen sich mindestens vier Punkte ausmachen, in denen diese Projekte radikaler ansetzen als Schleiermachers Kunstlehre des Verstehens.

(a) Quine fingiert eine hermeneutische Extremsituation, nämlich die Erstübersetzung einer völlig fremden Sprache, über die nicht das Geringste bekannt ist. Diese Situation unterscheidet die „radikale Übersetzung“ deutlich von der Auslegung literarischer Zeugnisse oder biblischer Texte.

(b) Zweitens wird angenommen, dass die Interpretationskunst nicht nur auf eine Theorie des Verstehens angewiesen ist, sondern auch in eine allgemeine Bedeutungstheorie integriert sein muss, die das Phänomen des sprachlichen Bedeutens problematisiert. Wie ist es überhaupt erklärlich, dass Lautketten und Tintenstriche, also physische Strukturen, auf etwas außerhalb ihrer selbst verweisen? Warum „bedeuten“ gewisse Strukturen überhaupt etwas und nicht vielmehr nichts? Die analytische Sprachphilosophie war im 20. Jahrhundert zentral mit der Frage befasst, was sprachliche Bedeutung überhaupt ist, also mit etwas, was man schon zu wissen glaubte, bis Wittgenstein und Quine viele tradierte Annahmen darüber in Zweifel zogen. Die Radikalisierung des Verstehensproblems durch eine Eingliederung in die Grundfragen der allgemeinen Bedeutungstheorie ist zugleich eine Tieferlegung der theoretischen Fundamente der Hermeneutik.

(c) Bei Quine mündet die Frage, was Bedeutung eigentlich ist, in die Auffassung, dass man das vermeintliche Phänomen des Bedeutens oder Meinens nicht unkritisch voraussetzen, sondern nur anerkennen dürfe, wenn man es auf weniger problematische Konzepte zurückgeführt hat. Quines „Bedeutungsskepsis“ besagt, dass die Sprachphilosophie ohne das Postulieren von Bedeutungen im Sinne von Intensionen auszukommen habe, unerlässliche semantische Begriffe seien allein Wahrheit und Bezugnahme.

(d) Von der Quineschen Bedeutungsskepsis zu unterscheiden und hermeneutisch interessanter ist der sinnkritische Gedanke, dass ein Sprecher keinen privilegierten Zugang zum Sinn seiner eigenen Äußerungen besitzt. Damit hat er auch keine Gewähr dafür, dass das, was er zu meinen scheint, sich überhaupt sinnvoll meinen lässt. Die Sinnkritik geht über die aus der Frühromantik bekannte Verstehensskepsis hinaus und stellt eine besonders interessante Herausforderung für eine Kunstlehre des Verstehens dar, die ja stets davon ausgeht, dass es etwas $z u$

und daß Verstehen auf jeden Punkt muß gewollt und gesucht werden.“ Hermeneutik (1819), KGA II/4, 127. 
verstehen gibt, also nicht ernsthaft damit rechnet, dass Verstehensbemühungen sich als Versuch am untauglichen Objekt erweisen könnten.

\section{Verstehensskepsis, Bedeutungsskepsis und Sinnkritik}

Der Zweifel daran, dass etwas vermeintlich Gemeintes überhaupt sinnvoll sagund meinbar ist, befindet sich außerhalb des Fragehorizonts der Aufklärungshermeneutik des 17. und 18. Jahrhunderts. Der sinnkritische Zweifel hat eine antimentalistische Pointe, die deutlich in einer Bemerkung Wittgensteins zum Ausdruck kommt: „Wenn man aber sagt: ,Wie soll ich wissen, was er meint, ich sehe ja nur seine Zeichen', so sage ich: Wie soll er wissen, was er meint, er hat ja auch nur seine Zeichen."“9

Husserl hatte von „bedeutungsverleihenden Akten“ des Bewusstseins gesprochen. Wittgensteins rhetorische Frage: „Aber ist es nicht unser Meinen, das dem Satz Sinn gibt?“10 mag darauf anspielen. Dieses Begründungsverhältnis zwischen privatem Meinen und öffentlichem Bedeuten kehrt Wittgenstein um: „Nur in einer Sprache kann ich etwas mit etwas meinen“. ${ }^{11}$ Die letztere Auffassung scheint Schleiermacher bis zu einem gewissen Grad zu teilen, denn er nimmt an, dass Denken grundsätzlich sprachförmig ist: „Man könnte sagen: die Rede wird mit dem Denken zugleich. Wir denken gar nicht eher, bis wir innerlich reden“. ${ }^{12}$ Das Formen eines Gedankens und das Bilden eines sprachlichen Ausdrucks wären demnach psychologisch keine separaten Vorgänge: „Das Denken wird durch innere Rede fertig und in so fern ist die Rede nur der gewordene Gedanke selbst ${ }^{\text {"13 }}$ Es fällt schwer, hier nicht an Kleists Formulierung von der „allmählichen Verfertigung der Gedanken beim Reden“ zu denken, die im selben Jahr niedergeschrieben wurde, in dem Schleiermacher erstmals seine Hermeneutikvorlesung hielt. Kleists erst posthum gedruckten Aufsatz konnte Schleiermacher nicht kennen.

In der traditionellen zweistufigen Semiotik von Platon bis Locke ging man demgegenüber davon aus, dass der Sprecher über bestimmte mentale Inhalte verfügt, für die er im zweiten Schritt ein Transportmedium braucht. Bei Locke sind

9 Ludwig Wittgenstein, Philosophische Untersuchungen, Frankfurt am Main 1971, § 504.

10 Ebd., $\S 358$.

11 Ebd., § 38. Searle hat später ein „Prinzip der Ausdrückbarkeit“ formuliert, demzufolge man „alles, was man meinen, auch sagen kann“. John R. Searle, Sprechakte (1969), Frankfurt am Main $1971,34$.

12 Kolleg 1819, Nachschrift Jonas, KGA II/4, 197.

13 Hermeneutik 1819, KGA II/4, 120. 
Vorstellungen Stellvertreter von Sachen, Wörter vertreten dann die Vorstellungen. Eine solche zweistufige Auffassung, in der das öffentliche Ausdrücken dem privaten Meinen folgt, abstrahiert von dem Umstand, dass auch die ausdrückbaren mentalen Inhalte schon in einem bestimmten Repräsentationsformat vorliegen, zu dem der Denker, folgt man Wittgenstein, keinen direkteren Zugang hat als seine Interpreten. Wenn man nur in einer Sprache etwas mit etwas meinen kann, geht das Meinen dem sprachlichen Bedeuten nicht voraus. So wendet Wittgenstein gegen die dem zweistufigen Paradigma folgende augustinische Spracherwerbstheorie ein, dass sie in Wirklichkeit von Wesen handle, die schon über eine Sprache verfügen und nun eine weitere erwerben - also vom Zweitsprachenerwerb. Augustinus schreibe über das lernende Kind, „als habe es bereits eine Sprache, nur nicht diese. Oder auch: als könne das Kind schon denken, nur noch nicht sprechen. Und ,denken' hieße hier etwas, wie: zu sich selber reden“ “. ${ }^{14}$

Die schwierige Frage, ob die These von der Sprachförmigkeit des Denkens für alle Denkinhalte plausibel ist oder nur für propositional verfasste Inhalte einer bestimmten Art und Komplexität, müssen wir hier auf sich beruhen lassen. Da Schleiermacher zweifellos eine Version der Sprachförmigkeitsthese vertritt ${ }^{15}$, ist es nicht diese These, in der Wittgensteins Sinnkritik das Verstehensproblem radikalisiert. Der entscheidende Radikalisierungsschritt besteht darin, dass nichts und niemand einen Sprecher davor schützt, in seinen sprachlichen Äußerungen die Grenzen des Sinns derart zu überschreiten, dass das Gesagte überhaupt keinen Gedanken mehr ausdrückt. Man kann zwar nicht denken, was sich nicht denken lässt, aber man kann etwas sagen, was keinen denkbaren Inhalt ausdrückt und insofern „einfach Unsinn“ ist. Im Vorwort des Tractatus hat Wittgenstein diese Asymmetrie eindrücklich beschrieben:

14 Wittgenstein, a.a.O., § 32. - Mir ist bewusst, dass es sich bei der Diagnose der mentalistischen Sprachauffassung sowohl bei Augustinus als auch bei Locke um problematische Vergröberungen handelt. Zu Augustinus vgl. Johann Kreuzer, Einleitung zu Augustinus, De Trinitate, Darmstadt 2001, XLVII - LI.; zu Locke vgl. Martin Lenz, Lockes Sprachkonzeption, Berlin/New York 2010, z. B. 532.

15 Nach Kimmerles Interpretation hat sich Schleiermacher in den beiden Akademiereden von 1829 von dem „Grundgedanken der Identität von Denken und Sprache abgewendet“ und nunmehr den „Prozeß des Heraustretens eines Gedankens in seine empirisch faßbare sprachliche Gestalt“ zum Gegenstand der Hermeneutik gemacht. Heinz Kimmerle, „Einleitung“ zu Friedrich Schleiermacher, Hermeneutik. Nach den Handschriften neu herausgegeben und eingeleitet von Heinz Kimmerle, Heidelberg 1974, 21. Zur Einschätzung der Identitätsthese und zum Verhältnis von Sprechen und Denken bei Schleiermacher vgl. Sarah Schmidt, Die Konstruktion des Endlichen: Schleiermachers Philosophie der Wechselwirkung, Berlin/New York 2005, 253-257. 


\begin{abstract}
„Das Buch will also dem Denken eine Grenze ziehen, oder vielmehr - nicht dem Denken, sondern dem Ausdruck der Gedanken: Denn um dem Denken eine Grenze zu ziehen, müßten wir beide Seiten dieser Grenze denken können (wir müßten also denken können, was sich nicht denken läßt). Die Grenze wird also nur in der Sprache gezogen werden können und was jenseits der Grenze liegt, wird einfach Unsinn sein.“16
\end{abstract}

Dass man, um dem Denken eine Grenze zu ziehen, beide Seiten dieser Grenze denken können muss, entspricht Hegels Figur der „Dialektik der Grenze“, derzufolge man eine Grenze in Gedanken schon überschritten haben muss, um sie zu bestimmen. Wittgenstein stellt nun fest, dass die Bestimmung der Grenzen des sinnvoll Sagbaren von dieser Dialektik nicht betroffen ist, weil sie „,von innen“ erfolgen kann. Er verweist auf die Asymmetrie, dass man zwar Undenkbares nicht denken, Unsinniges aber durchaus sagen kann. Man kann die Worte, die keinen denkbaren Inhalt ausdrücken, sehr wohl aussprechen, nur werden sie eben „Unsinn sein“.

Die antimentalistische Stoßrichtung der sinnkritischen Wende verändert die Geschäftsgrundlage einer dem mentalistischen Paradigma verhafteten Kunst des Verstehens. Wenn es keine „bedeutungsverleihenden Akte“ des Bewusstseins gibt, kein inneres „Meinen“, das unseren Worten Sinn verleiht, dann ist das mit einer Äußerung jeweils Gemeinte nicht mehr nur in der Hinsicht fragwürdig, dass es jeweils vom Interpreten ermittelt werden muss. Vielmehr ist es etwas von vornherein Problematisches, auch für den Sprecher selbst. Wenn die Bedeutung des Gesagten sich nicht aus dem inneren Meinen ergibt, dann stehen nicht mehr bloß die Möglichkeiten und Methoden des Verstehens auf dem Spiel, sondern der Sinn des Gesagten selbst. Eine Radikalisierung ist diese sinnkritische Wende, insofern klassische Verstehenstheorien selbst dort, wo sie skeptische Anwandlungen haben, nach den Grenzen des Verstehens fragen, nicht nach den Grenzen des Sinns. Der Unterschied lässt sich anhand einer berühmten Bemerkung von Humboldt illustrieren:

„Keiner denkt bei dem Wort gerade und genau das, was der andre, und die noch so kleine Verschiedenheit zittert, wie ein Kreis im Wasser, durch die ganze Sprache fort. Alles Verstehen ist daher immer zugleich ein Nicht-Verstehen, alle Uebereinstimmung in Gedanken und Gefühlen zugleich ein Auseinandergehen. “17

Humboldt lässt in seinem Hinweis auf die Grenzen des Verstehens - wie auch Schleiermacher in seinen Überlegungen zum Primat des Missverstehens - die privaten Denkinhalte des Sprechers unangetastet. Der Sprecher weiß sehr gut, was

16 Ludwig Wittgenstein, Tractatus logico-philosophicus, Frankfurt am Main 1960, Vorwort.

17 Wilhelm von Humboldt, „Ueber die Verschiedenheit des menschlichen Sprachbaues und ihren Einfluss auf die geistige Entwicklung des Menschengeschlechts“ (1830-35), in: ders., Gesammelte Schriften Bd. VII/1, Berlin 1907, 1-344, hier $64 \mathrm{f}$. 
er „bei dem Wort denkt“ und welchen „Gedanken und Gefühlen“ er Ausdruck verleihen will. Zum Problem werden nicht diese mentalen Daten, sondern allein deren „Übereinstimmung“ mit dem Interpreten.

Das, was der Sprecher bei einem Wort denkt, ist nach dem von Wittgenstein kritisierten intentionalistischen Modell vom sprachlichen Bedeuten die Quelle der Bedeutung. Die Äußerung eines Sprechers bedeutet nach diesem Modell, was der Sprecher meint, also auszudrücken beabsichtigt. Was die ,bedeutungsverleihenden Akte“ des Bewusstseins betrifft, so erfährt man bei Husserl nirgends genau, wie der Sprecher dieses „Verleihen“ von Bedeutung eigentlich bewerkstelligt. Erst Grice hat in den 60er und 70er Jahren des 20. Jahrhunderts eine Theorie dazu ausgearbeitet, die das Hervorgehen der Äußerungsbedeutung aus den Sprecherabsichten erklären soll. Eine Hauptschwierigkeit der intentionalistischen Semantik besteht darin, dass Absichten mit Gehalten, die spezifisch genug sind, um die ihnen in der Theorie zugedachte Rolle zu erfüllen, ihrerseits ein sprachförmiges Repräsentationsformat erfordern. In intentionalistischen Bedeutungstheorien ist der Repräsentationsgehalt sprachlicher Äußerungen von der Intentionalität mentaler Zustände abgeleitet, die selbst nicht problematisiert wird. Das ändert sich mit Wittgensteins Privatsprachenargument und mit Quines Bedeutungsskepsis. Was Wittgenstein und Quine verbindet, ist die Weigerung, in der Bedeutungstheorie bedeutungsverleihende mentale Tatsachen $\mathrm{zu}$ postulieren.

Verstehensskepsis kann man die Auffassung nennen, dass das Missverstehen der Normalfall und das Verstehen der glückliche und stets gefährdete Sonderfall ist. Die entsprechenden Tendenzen bei Schleiermacher und Friedrich Schlegel hat man „frühromantische Antihermeneutik“ genannt. Verstehensskepsis, so radikal sie sich etwa bei Derrida gerieren mag, betrifft ein Transportproblem, während Bedeutungsskepsis ein Problem mit dem Transportgut betrifft.

\section{Radikale Übersetzung}

Den Ausdruck „,radical translation“ hat Quine 1960 geprägt, um das Unternehmen der Erstübersetzung einer völlig fremden Sprache zu bezeichnen. ${ }^{18}$ Im zweiten Kapitel seines Hauptwerks Word and Object hat er dazu folgendes Gedankenexperiment ersonnen: Ein Sprachforscher trifft in einem entlegenen Winkel der Erde auf einen Stamm, der eine ihm völlig unbekannte Sprache spricht. Die Eingeborenen haben

18 Die folgende Darstellung orientiert sich an G. Keil, Quine, Stuttgart 2011, 37 - 51 und überschneidet sich mit meinem Artikel „Radikale Interpretation und radikale Übersetzung“, in: Handbuch Sprachphilosophie, hg.v. Nikola Kompa, Stuttgart 2015, 237 - 249. 
bisher isoliert von anderen Kulturen gelebt, so dass ihre Sprache niemals in eine andere Sprache übersetzt worden ist. Auch sonst ist nichts über diesen Stamm bekannt. Dem Feldlinguisten stellt sich nun die Aufgabe, die Laute, die die Eingeborenen von sich geben, in Äußerungen seiner eigenen Sprache zu übersetzen.

Gegenüber dem Normalfall des Übersetzens einer Äußerung oder eines Textes aus einer Quell- in eine Zielsprache gibt es hier eine zusätzliche Unbekannte, nämlich die Struktur der Quellsprache. Im gewöhnlichen Fall ist die Quellsprache bekannt, es gibt Wörterbücher, Grammatiken und Sammlungen von Idiomen, die der Übersetzer konsultieren kann. Hinsichtlich der „grammatischen“ Interpretation ist also schon vorgearbeitet. Schleiermacher ordnet der grammatischen Auslegung die „komparative“ Methode zu, bei der das zu Verstehende mit etwas Allgemeinem, bereits Verstandenem verglichen wird.

Der radikale Übersetzer befindet sich in einer ungünstigeren Lage. Er weiß über die Quellsprache noch nichts, so dass er auf der Basis derselben Belege sowohl genügend Informationen über die fremde Sprache als auch die Bedeutung der jeweiligen Äußerung ermitteln muss. Das Gedankenexperiment der radikalen Übersetzung ist ein Kunstgriff, durch den Quine den hermeneutischen Nullpunkt des Nichtverstehens fingiert. Diesen speziellen und artifiziellen Fall hatte Schleiermacher schlicht nicht vor Augen, wenn er postuliert: „[D]ie Mittheilung sezt auf jeden Fall die Gemeinschaftlichkeit der Sprache also eine gewisse Kenntniß derselben voraus. “19

Die Aufgabe des radikalen Übersetzers besteht nach Quine darin, ,aus den zu einem bestimmten Zeitpunkt beobachteten Reaktionen eines Menschen dessen derzeitige Sprache zu rekonstruieren." ${ }^{\text {20 }}$ Wie bekommt der Übersetzer einen Fuß in die Tür? Die ihm zur Verfügung stehenden Daten beschreibt Quine in behavioristischer Manier: „Die einzigen objektiven Daten, nach denen er sich richten kann, sind die Kräfte, die er auf die Außenflächen des Eingeborenen einwirken sieht, sowie das beobachtbare, stimmliche und sonstige Verhalten des Eingeborenen“. ${ }^{21}$

Eine erste Frage betrifft die Wahl einer geeigneten Übersetzungseinheit. Man übersetzt nicht Sprachen, sondern begrenzte Textstücke einer Sprache: Wörter, Sätze, Äußerungen. Der radikale Übersetzer geht von der Annahme aus, dass zwischen Äußerungen und außersprachlichen Gegebenheiten systematische Beziehungen bestehen. Gäbe es keine solchen Korrelationen, die er ausbeuten könnte, würde sein Unternehmen fehlschlagen. Diejenige sprachliche Einheit, die am besten auf solche Korrelationen hin untersuchbar ist, ist nach Quine der Satz.

19 Hermeneutik 1819, KGA II/4, 120.

20 W.V.O. Quine, Wort und Gegenstand (1960), Stuttgart 1980, 62.

21 Ebd. 
Diese Annahme entspricht dem Grundgedanken der Semantik der Wahrheitsbedingungen, den Wittgenstein so ausdrückt: „Einen Satz verstehen, heißt, wissen was der Fall ist, wenn er wahr ist“. ${ }^{22}$ Ein Interpret muss nicht wissen, ob der Satz wahr ist, um ihn zu verstehen, aber er muss wissen, unter welchen Bedingungen er wahr wäre. Man kann diesen Gedanken auch unter Verwendung des Begriffs des Glaubens reformulieren, denn etwas zu glauben bedeutet, es für wahr zu halten. Man versteht einen (Aussage-)Satz, wenn man weiß, welche Überzeugung der Sprecher damit ausdrücken möchte.

Die Auffassung, dass „Sätze der primäre Bedeutungsspeicher“23 sind, ist eine Abkehr von den Sprachtheorien der philosophischen Tradition, die ihren methodischen Ausgang vom Einzelwort genommen haben. Die These vom semantischen Primat des Satzes, die auf der beschriebenen Einsicht in die besondere Rolle der Aussagenwahrheit bei der Bestimmung von Bedeutungen beruht, mag befremdlich klingen, da Sätze schließlich aus Wörtern zusammengesetzt sind und da in natürlichen Sprachen die Bedeutungen von Sätzen systematisch mit denen ihrer Bestandteile zusammenhängen. Die kompositionale Struktur der Sprache widerspricht aber einer wohlverstandenen These vom semantischen Primat des Satzes nicht. Der frühkindliche Spracherwerb zeigt, dass die meisten der aus nur einem Wort bestehenden Äußerungen tatsächlich Sätze sind. Sagt das Kleinkind angesichts eines Hundes einsilbig „Hund“, so muss diese Äußerung als Einwortsatz interpretiert werden. ${ }^{24}$ Eine angemessene Übersetzung ins Erwachsenendeutsch mag lauten: „Dort ist ein Hund“ oder „Sieh mal, ein Hund!“. Im Spracherwerbsprozess zeigt sich der semantische Primat des Satzes nach Quine darin, dass „man Wörter nur lernt, indem man von ihren Rollen in bereits erlernten Sätzen abstrahiert“.25

Der Feldlinguist wird deshalb zunächst auf Äußerungen seines Gewährsmanns achten, die er für wahrheitsfähige Aussagen hält. Aber nicht alle Arten von Aussagen sind gleich gut geeignet. Um einen Fuß in die Tür zu bekommen, wird er im ersten Schritt auf möglichst kurze Äußerungen achten, die sein Gewährsmann angesichts einer plötzlichen, auffälligen, begrenzten Änderung einer Situation macht, in der sich beide gemeinsam befinden. Außerdem muss es sich um Äußerungen handeln, denen

22 Wittgenstein, Tractatus, a.a.O. (Anm. 16), 4.024.

23 W.V.O. Quine, Theorien und Dinge (1981), Frankfurt am Main 1985, 13.

24 Als Einwortsätze bezeichnen Linguisten elliptische Äußerungen wie „Feuer“ oder „Idiot“, die aus einem Wort bestehen, aber im jeweiligen Kontext als Sätze verstanden werden. Wenn „Idiot“ nicht natürlicherweise im Sinne von „Du bist ein Idiot“ verstanden würde, könnte man, wenn man das Wort in Anwesenheit eines Polizisten geäußert hat, ein Verfahren wegen Beamtenbeleidigung leicht abwenden.

25 Quine, Wort und Gegenstand, a.a.O. (Anm. 20), 101. 
man zustimmen kann, ohne über vorgängige Zusatzinformation zu verfügen. Quine nennt solche Äußerungen Beobachtungssätze (observation sentences). Eine brauchbare Situation wäre diese: Der Sprachforscher und sein Gewährsmann stehen nebeneinander und blicken schweigend in die Landschaft. Plötzlich hoppelt ein Kaninchen vorbei. Der Eingeborene sagt etwas, was wie „Gavagai“ klingt. Der Feldlinguist nimmt sein Notizbuch, notiert in der linken Spalte „Gavagai“ und in der rechten „Kaninchen“ oder „Sieh da, ein Kaninchen“, und zwar „als vorläufige, in weiteren Fällen zu erprobende Übersetzung““ ${ }^{26}$

Zur weiteren Erprobung seiner Übersetzungshypothese kann der Sprachforscher bei verschiedenen Gelegenheiten „Gavagai?“ fragen und achtgeben, ob sein Gewährsmann zustimmt oder nicht. Leider weiß er noch nicht, ob seine Hebung der Stimme als Frage interpretiert wird und welche Äußerungen oder Gesten er als Zustimmung oder Ablehnung werten kann. Um dies herauszubekommen, schlägt Quine vor, Äußerungen des Gewährsmanns nachzusprechen. Wenn man dadurch regelmäßig dasselbe kurze Wort auslöst, liegt die Vermutung nahe, dass es die Bedeutung von ,ja“ hat. Auch dies ist allerdings nicht mehr als eine Arbeitshypothese, die sich in weiteren Fällen bewähren muss. Quine verwendet viel Mühe und Phantasie darauf, sich mögliche Komplikationen bei der Überprüfung von Übersetzungshypothesen auszumalen.

Die logischen Verknüpfungen (nicht, und, oder, wenn ... dann) werden aus dem Satzzusammenhang heraus übersetzt. Stößt der radikale Übersetzer auf ein kurzes Wort, das jeden Beobachtungssatz, dem zuvor zugestimmt wurde, in einen verwandelt, der abgelehnt wird, so ist er auf die Negation gestoßen. Er hat ein behavioristisches Kriterium gefunden, das ihm das Recht gibt, das fragliche Wort mit „nicht“ zu übersetzen. Die Bedeutung der logischen Partikeln ergibt sich also als Nebenprodukt der radikalen Übersetzung der Sätze, in denen sie vorkommen. Dabei unterstellt der Übersetzer, dass es die logischen Verknüpfungen und die mit ihnen einhergehenden logischen Gesetze in der fremden Sprache gibt. Diese Unterstellung ist keine bloße Arbeitshypothese, da hier die Übersetzbarkeit der fremden Sprache überhaupt auf dem Spiel steht. Übersetzte man die Äußerungen eines Sprechers zum Beispiel so, dass sie eine große Zahl offener Widersprüche ausdrückten, dann würde es sehr schwierig, dem Sprecher überhaupt bestimmte Überzeugungen zuzuschreiben. Quine führt zur Illustration an, dass es auch in der muttersprachlichen Kommunikation vorkommt, dass jemand auf eine Frage mit „ja und nein“ antwortet. Wir nähmen aber in einem solchen Fall an, dass „der in Frage gestellte Satz bei der Affirmation und Negation jeweils verschieden gemeint ist; wir nehmen lieber das an, als daß der andere so dumm wäre, dasselbe $z u$

26 Ebd., 63. 
bejahen und $\mathrm{zu}$ verneinen“. ${ }^{27}$ Mit einer Äußerung konfrontiert, die, wörtlich übersetzt, den Satz vom Widerspruch verletzt, interpretieren wir wohlwollend, um diese Verletzung als scheinbare zu erweisen.

Die weiteren Schritte des Unternehmens der radikalen Übersetzung muss ich hier auf sich beruhen lassen. Quines Gedankenexperiment mündet in die These von der „Unbestimmtheit der Übersetzung“, aus der er sein Hauptargument für seine Bedeutungsskepsis gewinnt. Die Unbestimmtheitsthese ist aber für hermeneutische Fragestellungen, die um den Begriff des Verstehens zentriert sind, nicht von Belang.

\section{Das „principle of charity“}

Indem Quine den Feldlinguisten nach Möglichkeit widerspruchsvermeidend übersetzen lässt, bezieht er sich auf das in der analytischen Philosophie erstmals 1959 von Neil Wilson formulierte „principle of charity“. In der hermeneutischen Tradition hat dieses Prinzip zahlreiche Vorläufer. ${ }^{28} \mathrm{Zu}$ ihnen gehört das von Georg Friedrich Meier in seinem Versuch einer allgemeinen Auslegungskunst formulierte Prinzip der „hermeneutischen Billigkeit“ (aequitas hermeneutica). Meier versteht darunter „die Neigung eines Auslegers, diejenigen Bedeutungen für hermeneutisch wahr zu halten, welche mit den Vollkommenheiten des Urhebers der Zeichen am besten übereinstimmen, bis das Gegenteil erwiesen wird.“29 Schleiermacher kennt diese Interpretationsmaxime der Sache nach auch, hat aber keinen eigenen Namen dafür. Das Erscheinen eines Widerspruchs wertet Schleiermacher als Indiz für Nichtverstehen. Zu den „Maximen beim Verstehen“ gehöre diese: „Ich verstehe alles bis ich auf einen Widerspruch oder Nonsens stoße“. ${ }^{30}$ Auch Gadamers „Vorgriff auf Vollkommenheit“, den dieser als ein „Axiom aller Hermeneutik“31 ansieht, gehört in diese Reihe.

27 Ebd., 114.

28 Eine vergleichende Übersicht über diese Vorläufer (Dannhauer, Clauberg, Weise, Thomasius, Crusius, Meier, Lambert, Bolzano) verschafft Scholz, Verstehen und Rationalität, a.a.O. (Anm. 5), $35-67$.

29 Georg Friedrich Meier, Versuch einer allgemeinen Auslegungskunst, Halle 1757, § 39.

30 Zur Hermeneutik 1805 und 1809/10, KGA II/4, 6.

31 Hans-Georg Gadamer, Wahrheit und Methode. Grundzüge einer philosophischen Hermeneutik, Gesammelte Werke Bd. 1, Tübingen 1986, 376. - Einen erhellenden Vergleich der Prinzipien Meiers, Gadamers und Davidsons unternimmt Wolfgang Künne, „Prinzipien der wohlwollenden Interpretation“, in: Intentionalität und Verstehen, hg.v. Forum für Philosophie Bad Homburg, Frankfurt am Main 1990, 212-236. 
Bei Quine besagt das Prinzip der hermeneutischen Caritas, dass man bei der Übersetzung darauf achten sollte, dass die Aussagen des Sprechers sich nach Möglichkeit als widerspruchsfrei und wahr erweisen. Quine setzt das Prinzip also ein, um zu plausiblen Übersetzungen zu gelangen:

„Die Übersetzungsmaxime, die all dem zugrunde liegt, ist, daß Behauptungen, die oberflächlich auffällig falsch sind, auf verborgenen Unterschieden der Sprache beruhen. [...] Die durchaus vernünftige Annahme, die hinter dieser Maxime steckt, ist, daß die Dummheit des Gesprächspartners über einen bestimmten Punkt hinaus weniger wahrscheinlich ist als eine schlechte Übersetzung [...].“32

Quines Formulierung, dass niemand „so dumm wäre, dasselbe zu bejahen und zu verneinen“33, erfordert ihrerseits eine wohlwollende Interpretation. Eine solche Äußerung wäre nicht nur dumm, sondern ließe sich schwerlich noch als Ausdruck einer Überzeugung eines bestimmten Inhalts ansehen. Wenn jemand etwas und zugleich das Gegenteil davon glaubte, welchen Inhalt sollte seine Überzeugung haben? Nach den Regeln der klassischen Logik lässt sich aus einem Widerspruch Beliebiges ableiten (ex contradictione sequitur quodlibet). Deshalb zeigt die Zuschreibung offen widersprüchlicher Überzeugungen in der Regel an, dass mit der Übersetzung etwas nicht stimmt. Aristoteles hat sogar die These vertreten, dass das Prinzip vom ausgeschlossenen Widerspruch schon in den Umstand eingebaut sei, dass man überhaupt etwas mit seinen Worten meint. Sofern Worte überhaupt etwas bezeichnen, bezeichnen sie Bestimmtes. Wenn sie Beliebiges oder unbegrenzt Vieles bezeichneten, wäre die Möglichkeit vernünftiger Rede, ja selbst die des Denkens aufgehoben. ${ }^{34}$

Quine hat das „principle of charity“ auf die Übersetzung der logischen Verknüpfungen angewandt, wo es unverzichtbar ist. Man könnte es in einer Diktion, die Quine fremd ist, eine transzendentale Bedingung der Möglichkeit der Interpretation nennen. In den späteren Stadien der radikalen Übersetzung ändert das Prinzip seinen Charakter. Dort sinken Wahrheits- und Konsistenzunterstellungen zu widerrufbaren Hypothesen herab. Man probiert vorzugsweise Übersetzungen aus, die Wahrheit und Konsistenz maximieren, ist jedoch bereit, die Unterstellungen zurückzuziehen, wenn sich Gegenbelege türmen. Schließlich kommt es vor, dass Menschen Falsches oder versteckt Widersprüchliches glauben, und es ist nicht die Aufgabe des Übersetzers, tatsächlich bestehende Überzeugungsunterschiede zu verschleiern: „Eventually, of course, the linguist drops his initially

32 Quine, Wort und Gegenstand, a.a.O. (Anm. 20), 114-115.

33 Ebd., 114.

34 Vgl. Aristoteles, Metaphysik IV 4, 1006 b und XI 5, 1062 a. 
indispensable assumption that natives tell the truth. He does not go on forever modifying his growing theory of the native language so as to accomodate each succeeding affirmation as true“. ${ }^{35}$

Eine quasi-transzendentale Begründung des Caritas-Prinzips findet sich bei Davidson, der sich an Quines Rede von der „Unwahrscheinlichkeit“ der großen „Dummheit des Gesprächspartners“ stößt. Nach Davidson beruht die methodische Maxime, ,in einer Weise zu interpretieren, in der die Einigkeit optimiert wird, [...] nicht [...] auf einer nachsichtigen Voraussetzung mit Bezug auf die menschliche Intelligenz, die sich auch als falsch herausstellen könnte“. Vielmehr gelte:

„Wenn wir keine Möglichkeit finden, die Äußerungen und das sonstige Verhalten eines Geschöpfs so zu interpretieren, daß dabei eine Menge von Überzeugungen zum Vorschein kommt, die großenteils widerspruchsfrei und nach unseren eigenen Maßstäben wahr sind, haben wir keinen Grund, dieses Geschöpf für ein Wesen zu erachten, das rational ist, Überzeugungen vertritt oder überhaupt etwas sagt“. ${ }^{36}$

Bei oberflächlicher Lektüre läuft diese provokante Passage auf die ethnozentrische Behauptung hinaus, dass die Fremden entweder alles genauso sehen wie wir oder keine rationalen Wesen sind, ja nicht einmal Überzeugungen haben. Das klingt absurd - wenn man vergisst, dass die Pointe von Davidsons Überlegung darin besteht, dass dieser Fall zwischen menschlichen Sprechern nicht eintreten kann. Der radikale Übersetzer kann nur dann korrekt übersetzt haben, wenn er den größten Teil der fremden Äußerungen so interpretiert, dass er sie als Ausdruck derjenigen Überzeugungen ansehen kann, die in der gegebenen Situation zu haben nach den Maßstäben des Interpreten rational wäre. Ein hinreichend hoher Grad an Übereinstimmung ist nach Davidson Voraussetzung dafür, Überzeugungen allererst zu individuieren, und damit auch eine Bedingung der Möglichkeit der Interpretation. Überzeugungen werden ja über ihre Inhalte individuiert. Wenn nun eine Übersetzung oder Interpretation ergibt, dass einem Sprecher bizarre Überzeugungen über Kaninchen zugeschrieben werden müssen - wenn er zum Beispiel laut Übersetzung glaubt, dass Kaninchen große dickhäutige Tiere mit einem Rüssel sind ${ }^{37}$-, dann haben wir keinen guten Grund zu der Annahme, dass seine Äußerungen von Kaninchen handeln, und sollten eine andere Übersetzung ausprobieren. Je abwegigere Überzeugungen zu einem Redegegenstand die Inter-

35 W.V.O. Quine, „Philosophical Progress in Language Theory“, in: Metaphilosophy 1 (1970), 2 19, hier 17.

36 Donald Davidson, „Radikale Interpretation“ (1973), in: ders., Wahrheit und Interpretation, a.a.O. (Anm. 2) hier 199.

37 Vgl. mit einem anderen Beispiel Donald Davidson, „Sagen, daß“ (1968), in: ders., Wahrheit und Interpretation, a.a.O. (Anm. 2), 141 -162, hier $151 \mathrm{f}$. 
pretation ans Licht zu fördern scheint, desto wahrscheinlicher ist es, dass es sich überhaupt nicht um Überzeugungen über den fraglichen Gegenstand handelt, dass die Übersetzung also falsch ist und revidiert werden muss.

Freilich muss eine Übersetzung begrenzte Dissense zwischen Sprecher und Interpret enthüllen können. Beispielsweise könnte einer der beiden einem kaninchenbezogenen Aberglauben anhängen. Um diesen Überzeugungsunterschied allererst identifizieren zu können, müssen Interpret und Sprecher aber in unzähligen anderen Überzeugungen übereinstimmen. Der für Davidsons Begründung des Caritas-Prinzips entscheidende Punkt ist, dass „Meinungsverschiedenheit ebenso wie Meinungsgleichheit nur vor einem Hintergrund massiver Übereinstimmung verständlich sind“. ${ }^{38}$ Zusammenfassend:

„Je größer die Anzahl der Dinge, mit Bezug auf die jemand das Richtige glaubt, desto schärfer umrissen sind seine Irrtümer. Durch zu viele Fehler wird einfach der Brennpunkt unscharf. Die Interpretation wird demnach dadurch ermöglicht, daß wir die Möglichkeit massiven Irrtums a priori ausschließen können. Eine Interpretationstheorie, derzufolge jemand sehr viele falsche Sätze bejaht, kann nicht richtig sein [...].“39

\section{Radikale Interpretation beginnt zu Hause}

Unter „Übersetzen“ versteht man gemeinhin das Übertragen eines Textes einer Ursprungssprache in einen bedeutungsgleichen Text einer Zielsprache. Demgegenüber ist der Ausdruck „Interpretieren“ für Verstehens- und Deutungsprozesse im homophonen Fall reserviert. Interpretationsbedürftig können Äußerungen auch dann sein, wenn Sprecher und Interpret dieselbe natürliche Sprache sprechen. ${ }^{40}$

Der von Davidson verwendete Ausdruck „radikale Interpretation“ ist erklärtermaßen Quine verpflichtet, die beiden Projekte weichen aber in einigen Hinsichten voneinander ab. Zum einen meint Davidson, dass die Erstellung eines Quineschen „translation manual“, also eines zweisprachigen Übersetzungshandbuchs, dem Verstehensaspekt des Interpretierens zu wenig Rechnung trägt. Zum Beispiel könnte jemand wissen, dass „It is snowing“ eine Übersetzung von

38 Donald Davidson, „Radikale Interpretation“, a.a.O. (Anm. 2), 199.

39 Donald Davidson, „Denken und Reden“ (1975), in: ders., Wahrheit und Interpretation, a.a.O. (Anm. 2), 224-246, hier 244.

40 Der Unterschied ist im Englischen etwas geringer als im Deutschen; ein „interpreter“ ist ein Übersetzer, „to interpret“ heißt neben „auslegen, deuten, interpretieren“ auch „dolmetschen“. 
„Es schneit“ ist, ohne die Sätze zu verstehen. ${ }^{41}$ Ferner teilt Davidson den strengen Behaviorismus und die Intensionsskepsis Quines nicht, sondern weist auch den mentalen Einstellungen der Sprecher und Interpreten einen wesentlichen Ort in der Interpretationstheorie zu. Davon, dass Davidson das Caritas-Prinzip umfassender einsetzt und anders begründet als Quine, war bereits die Rede.

Davidsons Interpretationstheorie gehört in den Kontext seines Projekts einer wahrheitskonditionalen Bedeutungstheorie für natürliche Sprachen, einer Theorie, aus der alle Sätze der Form „S ist in L genau dann wahr, wenn p“ folgen, wobei $S$ für einen Satz der Objektsprache $L$ und $\mathrm{p}$ für einen Satz der Metasprache steht, der unter den gleichen Bedingungen wahr ist wie S. Unter einer Bedeutungstheorie versteht Davidson eine Theorie, deren Kenntnis einen Interpreten in die Lage versetzen würde, alle Äußerungen der fraglichen Sprache zu verstehen. Im interpretationstheoretischen Teil des Projekts geht es um die Frage, wie der Interpret sich in den Besitz der Belege bringt, die ihm das Verständnis ermöglichen und die Bedeutungstheorie bestätigen. „Radikal“ heißt das Interpretieren, weil eine Theorie für denjenigen Fall gesucht ist, in dem der Interpret weder die Bedeutungen der fremden Äußerungen noch die Überzeugungen und anderen mentalen Einstellungen der fremden Sprecher kennt.

An das Szenario des Quineschen Feldlinguisten knüpft Davidson auch deshalb nicht an, weil er annimmt, dass sich für die Verständigung zweier Sprecher derselben natürlichen Sprache ein strukturgleiches Problem stellt: „Die radikale Interpretation ist immer beteiligt, wenn man die Äußerungen eines anderen Sprechers versteht. “42 Diese Pointierung erklärt sich daraus, dass nicht von vornherein klar ist, was es heißt und woran man erkennt, ob zwei Sprecher dieselbe Sprache sprechen. ${ }^{43}$

Mit diesem Zug assimiliert Davidson das gewöhnliche Sprachverstehen an das Interpretieren und beide an das Übersetzen. Methodologisch gebe es keinen wesentlichen Unterschied zwischen Interpretieren und Übersetzen, da sich ein Hörer auch im homophonen Fall niemals sicher sein könne, dass der Sprecher mit

41 Man denke an John Searles Gedankenexperiment des „Chinesischen Zimmers“, in dem jemand, der nicht Chinesisch versteht, gleichwohl schriftlich gestellte chinesische Fragen mithilfe eines dicken Handbuchs, das syntaktische Regeln und mechanische Anweisungen enthält, in korrektem Chinesisch beantwortet.

42 Davidson, „Radikale Interpretation“, a.a.O. (Anm. 2), 183.

43 „Das Problem der Interpretation gilt für die Muttersprachen ebenso wie für Fremdsprachen. Für Sprecher derselben Sprache stellt es sich in Form der Frage: Wie läßt sich feststellen, daß die Sprache dieselbe ist?“ (ebd.). Quine hatte das allerdings auch schon bemerkt: „Now it should be noted that [...] the resort to a remote language is not really essential. On deeper reflection, radical translation begins at home“. W.V.O. Quine, Ontological Relativity and Other Essays, New York 1969, 46. 
seiner Äußerung das meint, was er selbst mit einer gleichlautenden Äußerung meinen würde. In einem seiner späten Aufsätze behauptet Davidson dann bündig, dass keine zwei Sprecher jemals dieselbe Sprache sprächen und dass es so etwas wie eine Sprache nicht gebe. ${ }^{44}$ Diese Behauptung ist eine rhetorische Übertreibung, die der Plausibilisierung bedarf. Sprechen zwei Sprecher des Deutschen nicht dieselbe natürliche Sprache? Nun, beide sprechen deutsch, aber es sprechen nicht beide dasselbe Deutsch. Beispielsweise sprechen Teilnehmer einer sprachphilosophischen Konferenz einen Soziolekt des Deutschen, der sich unter anderem dadurch auszeichnet, dass er die Vokabel „Soziolekt“ enthält, ein Wort, das die Mehrheit der Sprecher des Deutschen nicht kennt. Zusätzlich spricht jeder einzelne Sprecher einen Idiolekt des Deutschen, eine höchst individuelle Variante der deutschen Gemeinsprache. Davidson illustriert das Phänomen des Idiolekts am Phänomen der Malapropismen, sprachlicher Fehlleistungen halbgebildeter Sprecher, die an Stelle des korrekten Fremdwortes ein phonetisch ähnliches verwenden, nicht ahnend, dass sie etwas falsch gemacht haben. Beispiele bietet Frau Stöhr in Thomas Manns Zauberberg. ${ }^{45}$ Auch Interviews mit Fußballern sind eine Fundgrube für Malapropismen: „Das wird alles von den Medien hochsterilisiert“ (Bruno Labbadia); „Wir sind eine gut intrigierte Truppe“ (Lothar Matthäus); „Ich habe ihn nur ganz leicht retuschiert“ (Olaf Thon).

Hans Castorp leidet, wenn Frau Stöhr zur Rede anhebt, aber sie wird stets verstanden. Wir sind schon hinsichtlich dessen, was Schleiermacher die „grammatische“ Interpretation nannte, derart geübte Interpreten, dass wir viele Fehler und idiolektale Abweichungen von der Sprachnorm nicht einmal bemerken, sondern stillschweigend korrigieren. Studierenden der Sprachwissenschaft, die zum ersten Mal eine Transkription von gesprochener Sprache anfertigen müssen, sind in der Regel verblüfft. Mündliche Äußerungen auch kompetenter Sprecher enthalten eine Vielzahl mehr oder weniger gravierender Verstöße gegen grammatische und semantische Regeln, Morphologie und Phonetik ihrer Muttersprache: Sie sind durchsetzt von Anakoluthen, Ellipsen, Antizipationen, Versprechern, Vertauschungen, Missgriffen in der Wortwahl etc. Selbst bei akademisch gebildeten Sprechern sind in frei formulierter

44 „I conclude that there is no such thing as a language, not if a language is anything like what many philosophers and linguists have supposed. [...] We must give up the idea of a clearly defined shared structure which language-users acquire and then apply to cases.“ Donald Davidson, „A Nice Derangement of Epitaphs“, in: Truth and Interpretation. Perspectives on the Philosophy of Donald Davidson, hg.v. E. Lepore, Oxford 1986, 447 - 458, hier 446.

45 „Karoline Stöhr war entsetzlich. [...] Sie sagte ,Agonje“ statt Todeskampf; ,insolvent‘, wenn sie jemandem Frechheit zum Vorwurf machte [...]. Mit den liegenden Schneemassen, sagte sie, sei es ,eine wahre Kapazität“ [...].“ Thomas Mann, Der Zauberberg. Große kommentierte Frankfurter Ausgabe, Abt. I, Bd. 5.1, Frankfurt am Main 2002, $451 \mathrm{f}$. 
gesprochener Sprache die syntaktisch korrekten Sätze in der Minderheit. Der Verstoß gegen die Regeln der deutschen Standardsprache ist kein exotischer Sonderfall, sondern der Normalfall. Manche Abweichungen von der Sprachnorm geschehen auch absichtlich, beispielsweise Wortspiele, innovative Metaphern oder absichtliche Malapropismen (,zum Bleistift“).

Davidson bilanziert, dass „there is no such thing as a language“, wenn man unter einer Sprache das verstehe, was viele Philosophen und Linguisten unter ihr verstanden haben, nämlich ein klar umrissenes konventionelles Regelwerk, das Sprachbenutzer erlernen und auf einzelne Fälle anwenden und dessen Befolgung zur erfolgreichen Kommunikation unerlässlich ist. Freilich gebe es natürliche Sprachen, beispielsweise das Deutsche, das aber strenggenommen aus einer großen Menge von Privatsprachen, nämlich idiolektaler Varianten des Deutschen bestehe bzw. sich in ihnen realisiere. ${ }^{46}$ Die Vorstellung, dass gelingende Verständigung sich unter Rekurs auf konventionelle Regeln erklären ließe, müsse jedenfalls aufgegeben werden. ${ }^{47}$

\section{Die Rolle der Sprecherabsichten}

Erklärungsbedürftig ist, warum die erwähnten Regelverstöße und idiolektalen Eigenheiten so selten die Interpretation des Gesagten gemäß dem intendierten Sinn vereiteln. Da die Erklärung durch eine angeblich geteilte Sprache zu kurz greift, müssen wir unser Augenmerk auf die involvierten Fähigkeiten der Sprecher und Hörer richten, und zwar auf diejenigen in Verständigungssituationen aktualisierten Fähigkeiten, für deren Erwerb man sich nicht theoretisch mit Hermeneutik oder Interpretationstheorie beschäftigt haben muss. Davidsons Erklärung ${ }^{48}$ lautet in skizzenhafter Form wie folgt:

Diesseits des Quineschen Szenarios des radikalen Übersetzers in der Fremde haben Interpreten stets bestimmte Erwartungen und Vorannahmen über das Sprachverhalten des Sprechers. Davidson nennt diese Annahmen die „Ausgangstheorie“ des Hörers (prior theory). Sagt nun ein Sprecher etwas leicht Irri-

46 „Of course I don’t deny that there are languages like English, German and Basque or that, in some ordinary sense, people speak them. What is clear to almost everyone, however, is that no two speakers speak in exactly the same way; each speaker has, as Chomsky has maintained, a personal idiolect.“ Donald Davidson, „Reply to Kemmerling“, in: R. Stoecker (Hg.): Reflecting Davidson. Donald Davidson Responding to an International Forum of Philosophers, Berlin und New York 1993, 117.

47 Vgl. Davidson, „A Nice Derangement of Epitaphs“, a.a.O. (Anm. 44), 446.

48 Vgl. ebd., $441-446$. 
tierendes, unterläuft ihm beispielsweise ein Malapropismus, so veranlasst dies den Interpreten, seine Ausgangstheorie im Lichte des Gehörten und unter Anwendung des Caritas-Prinzips blitzschnell zu einer „Übergangstheorie“ (passing theory) zu modifizieren, die den neuen Belegen Rechnung trägt und die Interpretation gemäß der Sprecherintention ermöglicht. Wollte man das implizite Räsonnement des Hörers verbalisieren, so könnte es lauten: „Aha, mit ,mariniert“ scheint er ,manieriert‘ zu meinen. Dann ergibt das Gesagte einen Sinn. Nun gut, ich bin ein höflicher Mensch und sag’s ihm später oder gar nicht.“

Versetzen wir uns nun in die Perspektive des Sprechers. Während der Hörer erst im Zuge der Interpretation die Sprecherüberzeugungen ermittelt, muss der Sprecher sich nicht fragen, was er glaubt und welcher Überzeugung er mit seinen Worten Ausdruck verleihen will. Man kann diese Asymmetrie zwischen Selbstund Fremdzuschreibung so ausdrücken, dass der Sprecher eine hermeneutische Autorität bezüglich dessen hat, was ein Wort in seinem Mund bedeutet oder bedeuten soll. ${ }^{49}$ Das gilt auch, wenn er das Wort absichtlich oder unabsichtlich in von der Sprachnorm abweichender Weise verwendet. Auch Frau Stöhr weiß im Normalfall, was sie sagen will, und sie meint mit ihren Worten das, was sie zu meinen beabsichtigt. ${ }^{50}$ Der Interpret hat dieses jeweils Gemeinte zu ermitteln. Auch wenn der Sprecher wissentlich oder unwissentlich Regeln verletzt, hat der Hörer den Sprecher gemäß dessen Absicht zu interpretieren.

Was nun den Begriff der Bedeutung betrifft, so kehrt der späte Davidson in behutsamer Annäherung an die intentionalistische Semantik die Explikationsrichtung zwischen sprachlichen Konventionen und der erfolgreichen Übermittlung von Absichten um. Allgemein könne man den Begriff der Bedeutung nur vom Erfolgsfall der gelungenen Verständigung her aufklären und diesen vom Verständnis der Sprecherabsichten her:

„Der Begriff der Bedeutung basiert vollständig auf Fällen erfolgreicher Kommunikation [...], in denen eine Person beabsichtigt, auf eine bestimmte Weise verstanden zu werden, und so verstanden wird. [...] Was sollen wir sagen, was der Sprecher meint, wenn wir uns vom Erfolgsfall entfernen? Ich denke, es gibt keine klare Antwort auf diese Frage.“51

49 Vgl. dazu Donald Davidson, „First Person Authority“ (1984), in: ders., Subjective, Intersubjective, Objective, Oxford 2001, 3-14, hier 12-13.

50 Absichten, mit seinen Worten Bestimmtes zu meinen, nennt Davidson „semantic intentions“. Vgl. Donald Davidson, „Locating Literary Language“, in: Literary Theory After Davidson, hg.v. R.W. Dasenbrock, University Park, PA 1993, 295 -308, hier 299.

51 Donald Davidson und Kathrin Glüer, „Bezüge und Übergänge. Ein Interview mit Donald Davidson“, in: Glüer, Davidson, a.a.O. (Anm. 1), 153-176, hier 166. 
Davidson vertritt also die Auffassung, dass die Bedeutung sprachlicher Äußerungen nichts ist, was man mithilfe von Wörterbüchern und Regelwerken ermitteln kann, sondern dasjenige, worin Sprecher und Hörer in gelungener Verständigung übereinstimmen. Er übernimmt von der intentionalistischen Theorie den Gedanken, dass die Interpretation gemäß der Sprecherabsicht eine notwendige Bedingung für sprachliche Verständigung ist. Er übernimmt aber nicht die weitergehenden Gedanken, dass man Sprecherabsichten benutzen kann, um Bedeutung zu definieren oder auch nur als Belegmaterial für Bedeutungszuschreibungen zu verwenden. ${ }^{52}$ Und erst recht vertritt er nicht die These der einsinnigen Abhängigkeit der sprachlichen Bedeutung vom privaten Meinen, die man in Anlehnung an eine Figur in Lewis Carrolls Alice im Wunderland eine „Humpty Dumpty“-Theorie der Bedeutung nennt. Darunter wird die Auffassung verstanden, dass ein Sprecher seine Worte von Fall zu Fall bedeuten lassen kann, was ihm gerade beliebt. ${ }^{53}$ Eine Humpty Dumpty-Auffassung der Bedeutung ist eine Karikatur der „bedeutungsverleihenden Akte“ Husserls.

Sehen wir genauer hin. Humpty Dumpty kann nur deshalb bedeutungsverleihende Absichten haben, weil er eine hermeneutische Autorität in Bezug auf das hat, was er seine Worte bedeuten lassen will. Er weiß, was die Worte in seinem Mund bedeuten sollen. Dies aber kann er nur wissen, wenn er schon eine Sprache hat: ein hinreichend differenziertes symbolisches Ausdrucksmedium, in dem seine eigenen intentionalen Zustände in einer für ihn verständlichen Weise repräsentiert sind. Wittgensteins wohlverstandenes Diktum „Nur in einer Sprache können wir etwas mit etwas meinen“"54 gilt auch für Humpty Dumpty. Während Wittgenstein aber unter einer Sprache eine öffentliche regelgeleitete Praxis versteht, veranschlagt Davidson die Rolle von Regeln geringer. Kreativer Sprachgebrauch, mit dem ein Sprecher die konventionellen Regeln einer Sprache verletzt, ist stets möglich, ohne dass der Sprecher eo ipso uninterpretierbar würde. Die Wahrheit scheint in der Mitte zwischen einem konventionalistischen Regelbefolgungsmodell der Sprache und dem intentionalistischen Humpty Dumpty-Modell zu liegen. Genauer: Es muss etwas geben, was den wahren Kern beider Ideen bewahrt, die Extreme vermeidet und den Widerspruch zwischen ihnen als scheinbaren erweist.

52 „Die Hauptschwierigkeit ist [...], daß keine Aussicht besteht, der Zuschreibung fein unterschiedener Intentionen unabhängig von der Interpretation der gesprochenen Sprache Sinn beizulegen.“ Davidson, „Radikale Interpretation“, a.a.O. (Anm. 2), 186.

53 „,When I use a word', Humpty Dumpty said, in rather a scornful tone, ,it means just what I choose it to mean - neither more nor less. “" Lewis Carroll, Through the Looking-Glass, Raleigh, NC 1872, 72.

54 Wittgenstein, Philosophische Untersuchungen, a.a.O. (Anm. 9), § 38. 
Nach Davidson besteht die Lösung darin, dass der Sprecher neben der semantischen Absicht, etwas Bestimmtes mit seinen Worten zu meinen, noch eine weitere Absicht hat, nämlich die, gemäß seiner Absicht interpretiert zu werden. Ein kooperativer Sprecher möchte verstanden werden, und weil er diese Absicht hat, gibt es für das, was er mit seinen Worten überhaupt meinen kann, Restriktionen. Wenn er weiß, dass seine privatsprachliche Äußerung nicht die wundersame Kraft hat, dem Hörer Verständnis einzupflanzen, kann er dies auch nicht beabsichtigen. Die entscheidende, bei Davidson nur angedeutete Überlegung betrifft den Begriff der Absicht: Da man etwas, was man selbst für unmöglich hält, nicht beabsichtigen kann - das liegt im Begriff des Beabsichtigens -, muss der idiolektale Sprecher der Überzeugung sein, dass der Hörer in der Lage ist, das Gesagte gemäß der Sprecherabsicht zu verstehen. Der Sprecher muss, wie es bei Schleiermacher heißt, von vornherein „Rüksicht [...] auf die Gedankenverbindung und das Verstehen des Hörenden“ nehmen. Grundsätzlich könne „nichts so gemeint sein wie es die Hörer unmöglich haben verstehen können“. 55

Eben dies ist bei kompetenten Sprachbenutzern der Fall: Wir verwenden eine paradoxe Formulierung, eine Ellipse, einen Neologismus, Ironie, eine Metapher genau dann, wenn wir annehmen, dass die Äußerung verstehbar ist, wenn wir also davon ausgehen dürfen, dass die Hörer im Besitz der Fähigkeiten und Mittel sind, das jeweils Gemeinte zu ermitteln. Auch der Sprecher hat nämlich eine „prior theory“. Sie besteht in seinen Überzeugungen über die Ausgangstheorie des Interpreten, also in dem, was er über des Interpreten Erwartungen hinsichtlich seines, des Sprechers, Sprachgebrauch annimmt. ${ }^{56}$ Manchmal irrt sich der Sprecher in diesen Annahmen und überschätzt die Fähigkeiten des Interpreten. Manchmal hat der Sprecher auch die Aufgabe, dem Interpreten die für das Verständnis benötigten „clues“ erst zu verschaffen, etwa durch Zusatzinformationen, Deixis, Ironiesignale etc.

Des Interpreten Übergang von der Ausgangstheorie zur Übergangstheorie ist selbst nicht regelgeleitet und auch nicht über Bedeutungskonventionen vermittelt. Die Entwicklung der „passing theory“ ist eine nichttriviale Aufgabe, die ,wit, luck, and wisdom ${ }^{“ 57}$ erfordert. In dieser Bemerkung Davidsons mag man ein fernes Echo des „divinatorischen“ Momentes des Verstehens vernehmen. Als ein „Erahnen“ oder „Erraten“ ist dieses Verfahren aber fehl- oder unterbestimmt, denn die fragliche Praxis mag regellos sein, aber sie ist nicht vernunftlos. Die prompte Entwicklung einer

55 Zur Hermeneutik 1805 und 1809/10, KGA II/4, 27. Für die Auslegung aus historischer Distanz bedeutet dies, dass der Interpret sich in den ursprünglichen Adressaten zurückzuversetzen hat: „Man muß suchen der unmittelbare Leser zu werden um Anspielungen zu verstehen [...].“ Ebd., 8. 56 Vgl. Davidson, „A Nice Derangement of Epitaphs“, a.a.O. (Anm. 44), 442.

57 Ebd., 446. 
Übergangstheorie erfordert ein explizites oder implizites Räsonnement des Hörers darüber, was der Sprecher vernünftigerweise gemeint haben kann.

Wenn diese Skizze im Wesentlichen richtig ist, kann kreativer Sprachgebrauch auch bedeutungskonstitutiv sein, ohne dass man eine Humpty-Dumpty-Theorie der Bedeutung akzeptieren müsste. Was ein Sprecher seine Worte bedeuten lassen kann, ist nicht beliebig. Die Restriktionen bestehen aber nicht in einem System verbindlicher Bedeutungskonventionen, sondern in der vom Sprecher zu gewährleistenden Interpretierbarkeit, für die von Fall zu Fall andere Bedingungen erfüllt sein müssen. Wie weit Idiolekte einander überlappen müssen, um Interpretierbarkeit zu gewährleisten, ist am grünen Tisch nicht zu entscheiden.

Wittgenstein fragt rhetorisch „Kann ich mit dem Wort ,bububu' meinen ,Wenn es nicht regnet, werde ich spazieren gehen'?"58 Dies scheint nicht ohne weiteres möglich zu sein. Die Erklärung dafür ist allerdings aus Sicht des späten Davidson nicht, dass „bububu“ nun einmal nicht die fragliche Bedeutung hat, sondern dass der Sprecher nicht ohne weiteres beabsichtigen kann, mit „bububu“ das Fragliche zu meinen. ${ }^{59}$ Die Bedingung, dass der Sprecher seine Äußerung für interpretierbar halten muss, wird leicht übersehen, was daran liegt, dass sie in die Absicht, einen Inhalt zu kommunizieren, schon eingebaut ist: Man kann nur beabsichtigen, was man für im Prinzip durch eigenes Zutun erreichbar hält. (Darin unterscheidet sich das Beabsichtigen vom Wünschen und vom Hoffen.)

In Quines Szenario der radikalen Übersetzung fehlen übrigens die Sprecherabsichten, was es in einer wichtigen Hinsicht unvollständig macht und seine Erklärungskraft schwächt. Das Gedankenexperiment beschreibt Kommunikationssituationen, ist aber undialogisch konstruiert und verbleibt in der Beobachterperspektive: Der Feldlinguist versucht sich einen Reim auf die Äußerungen des Gewährsmanns zu machen, ohne einen Gedanken darauf zu verschwenden, dass es sich um Sprecher handelt, die kommunikative Absichten verfolgen und deren Gesprächsbeiträge plausiblerweise darauf zielen, verstanden zu werden, und sei es recht und schlecht. Bezieht man nun zusätzlich die Überzeugungen ein, die mit den Sprecherabsichten analytisch verbunden sind und die Sprecher einander wechselseitig unterstellen müssen, so ergibt sich schon in der vermeintlich einfach strukturierten Gavagai-Situation ein komplexeres und hermeneutisch interessanteres Bild als das von Quine gezeichnete. ${ }^{60}$

58 Wittgenstein, Philosophische Untersuchungen, a.a.O. (Anm. 9), § 38.

59 Es mögen sich Kontexte fingieren lassen, in denen er diese Absicht haben kann, deshalb ist die Einschränkung „nicht ohne weiteres“ am Platze.

60 Gegen Quine ist in diesem Sinne eingewandt worden, dass er den dialogischen Charakter der Interaktion zwischen Feldforscher und Gewährsmann verkenne und zudem eine Reihe von stillschweigenden hermeneutischen und anthropologischen Voraussetzungen mache, die seinen 


\section{Ausblick: Woran bemisst sich Verstehenserfolg?}

„Fälle erfolgreicher Kommunikation“ nennt Davidson solche, „in denen eine Person beabsichtigt, auf eine bestimmte Weise verstanden zu werden, und so verstanden wird“. ${ }^{61}$ Eine Kommunikation ist nach dieser Auffassung genau dann erfolgreich, wenn der Sprecher gemäß seiner Absicht verstanden wird. In der Hermeneutiktradition wird bekanntlich kontrovers diskutiert, ob man bei diesem Erfolgsstandard stehen bleiben soll und wie er sich zu weiteren möglichen Verstehenszielen verhält. Nach Schleiermachers berühmter Formulierung besteht die Aufgabe des Interpreten darin, „die Rede zuerst ebenso gut und dann besser zu verstehen als ihr Urheber“. ${ }^{2}$ Den zweiten Teil der Aufgabe begründet Schleiermacher damit, dass „wir vieles zum Bewußtsein zu bringen suchen [müssen] was ihm unbewußt bleiben kann außer sofern er selbst reflectirend sein eigener Leser wird“. ${ }^{63}$

Die Zusatzaufgabe des Besserverstehens, die Schleiermacher eine „unendliche“64 nennt, ist ein Thema für sich, das den Rahmen dieses Beitrags sprengen würde. Was das von Davidson genannte Ziel betrifft, einen Sprecher so zu verstehen, wie er verstanden zu werden beabsichtigt, so stellt sich die Frage, woran sich diese Übereinstimmung bemisst und wie man feststellen kann, ob sie erreicht ist. Werfen wir einen vergleichenden Blick auf Schleiermachers Formulierung der Übereinstimmungsbedingung. Schleiermacher spricht an verschiedenen Stellen von einer Identität der Gedanken des Sprechers und des Hörers. Sprecher wie Hörer hätten das Ziel, dass beide dasselbe denken; insofern sei

„die Auslegung nichts als die Umkehr der Redekunst. [...] Durch das Verstehen soll aus dem Reden wieder ein Denken werden. [...] [D]as Bestreben, daß das Denken in dem einen ein Denken in allen werden soll, ist eben in der Rede gegeben.“

Der Redende wie der Verstehende haben das Ziel, dass „,der Gedanke des einen mit dem des andern identisch werde“. ${ }^{65}$

Ein zweites von Schleiermacher genanntes Verstehensziel ist die Übereinstimmung in den Gefühlen. Man findet es etwa in seiner Darstellung der Leistung des

Anspruch des „starting from scratch“ Lügen strafen. Vgl. etwa Hans-Johann Glock, Quine and Davidson on Language, Thought and Reality, Cambridge 2003, 175-182.

61 Davidson und Glüer, „Bezüge und Übergänge“, a.a.O. (Anm. 51), 166.

62 Hermeneutik 1819, KGA II/4, 128.

63 Ebd.

64 Ebd., 129.

65 Kolleg 1819, Nachschrift Jonas, KGA II/4, 197. 
charismatischen religiösen Redners. Der „geübte Sinn der Gemeine begleitet überall den seinigen“ und die Wirkung der Rede ist im Erfolgsfall, dass „sein Herz und das eines Jeden nur der gemeinschaftliche Schauplatz deßelben Gefühls“ werden. ${ }^{66}$

Dieselben Maßstäbe wie Schleiermacher nennt auch Humboldt, nämlich die „Uebereinstimmung in Gedanken und Gefühlen“ zwischen Sprecher und Hörer, wenn auch im Modus des unerreichbaren Ideals. In der oben bereits zitierten Passage heißt es:

„Keiner denkt bei dem Wort gerade und genau das, was der andre [...]. Alles Verstehen ist daher immer zugleich ein Nicht-Verstehen, alle Uebereinstimmung in Gedanken und Gefühlen zugleich ein Auseinandergehen.“ ${ }^{67}$

Humboldts Auffassung ist also, dass (a) verschiedene Sprecher unterschiedliche Konnotationen mit den gleichen Worten verbinden, dass (b) diese Unterschiede zum partiellen Nichtverstehen führen und dass (c) dieses Verstehensdefizit darin besteht, dass Sprecher und Hörer nur unvollkommen in ihren mit den jeweiligen Konnotationen verbundenen Gedanken und Gefühlen übereinstimmen.

Ich möchte mich abschließend der Frage zuwenden, ob die vollkommene Übereinstimmung in Gedanken und Gefühlen als angemessener Erfolgsmaßstab für eine erfolgreiche Interpretation bzw. für gelungene sprachliche Verständigung gelten kann. Zunächst liegt auf der Hand, dass sich gravierende Verifikationsprobleme stellen. Wie können Sprecher und Hörer feststellen, ob der Zustand der Übereinstimmung erreicht ist? Woher kann Humboldt wissen, dass er in einem gegebenen Fall nicht erreicht ist? Als Interpreten sprachlicher Gebilde hören oder lesen wir die Worte eines anderen, die wir auf dem Hintergrund unseres Vorwissens bestmöglich zu verstehen suchen. Wir können einander tief in die Augen blicken und bedeutungsschwer sagen „Ich verstehe Dich“, aber da die mit den Worten verbundenen Konnotationen divergieren, bleibt nach Humboldt dem vermeintlichen Verstehen stets ein tieferes Missverstehen beigesellt. Die zitierte Passage macht deutlich, dass Humboldt hier keine identifizierbaren, behebbaren Missverständnisse im Sinn hat, sondern eine unaufhebbare wechselseitige Intransparenz der Sprecher - die es dann aber zugleich unmöglich machen müsste, sie begründet zu diagnostizieren.

Schleiermacher nimmt demgegenüber an, dass im Falle einer engen Geistesoder Seelenverwandtschaft zwischen Sprecher und Hörer die Möglichkeit eines „unmittelbaren Verstehens“ bestehe und dass in solchen Fällen die Rede auch nicht der Auslegung bedürfe:

66 Über die Religion. Reden an die Gebildeten unter ihren Verächtern (1799), KGA I/2, 269. 67 Humboldt, a.a.O. (Anm. 17), $64 \mathrm{f}$. 
„[W]enn ich mit einem so stehe, daß meine Rede nichts ist als der gewordene Gedanke selbst, daß ich mich gleich in ihn hineindenke durch meine Rede, da ist keine Auslegung nöthig, sondern da wird die Rede so unmittelbar verstanden, wie sie unmittelbar der gewordene Gedanke selbst ist.“68

Das „unmittelbare“ Verstehen durch Empathie ist nichts anderes als die erfolgreiche Anwendung der divinatorischen Methode: „Die divinatorische [Methode] ist die welche indem man sich selbst gleichsam in den andern verwandelt, das Individuelle unmittelbar aufzufassen sucht.“69

Außerhalb des Paradigmas der Schleiermacherschen „Einfühlungshermeneutik“, wie Gadamer sie despektierlich genannt hat, wird man nach anderen Maßstäben dafür suchen, ob und in welchem Umfang Verstehen erreicht ist.

Flüssige Kommunikation ist sicherlich kein Beleg für erfolgreiche Verständigung. Der bloße Umstand, dass eine Konversation mühelos mit Rede und Gegenrede voranschreitet, beweist wenig. Menschen lachen oft über Witze, die sie nicht verstanden haben; manchmal ist ihnen bewusst, dass sie etwas nicht verstanden haben, manchmal nicht. Auch das sprichwörtliche Aha-Erlebnis kann trügerisch sein. Oft zeigt es plötzliches Verstehen an, aber es garantiert es nicht. Allgemein scheinen psychologische Kriterien ungeeignete Erfolgsmaßstäbe für Verstehen $\mathrm{zu}$ sein, denn es ist stets möglich, dass ein Interpret verstanden $\mathrm{zu}$ haben glaubt, ohne verstanden $\mathrm{zu}$ haben.

Wenden wir den Blick in eine andere Richtung. Zu den Aufgaben von Bildungseinrichtungen gehört es, das Textverständnis von Schülern und Studierenden zu überprüfen. Dabei geben sie sich mit der Versicherung, man habe etwas verstanden, aus gutem Grund nicht zufrieden: nicht (nur), weil sie die Wahrhaftigkeit der Befragten nicht voraussetzen können, sondern weil auch die aufrichtige Versicherung, man habe etwas verstanden, das Verständnis nicht beweist. Verstehenserfolg bemisst sich schlicht nicht daran, ob sich ein bestimmtes Erfolgsgefühl einstellt. Was machen Lehrer tatsächlich, um das Textverständnis von Schülern zu überprüfen? Sie stellen Fragen zum Text. Je besser diese beantwortet werden, desto besser ist der Text verstanden. Eine zweite Methode der Wahl ist es, den Text in eigenen Worten wiedergeben zu lassen. Wer eine schwierige Passage bei Kant in eigenen Worten paraphrasieren kann, hat sie besser verstanden als der, der sie nur in Kants Diktion reproduzieren kann.

Aus Humboldts und Schleiermachers Sicht müssen diese Verfahren der Verständnissicherung, die das Verstehen unter Preisgabe des Ziels der „Übereinstimmung in Gedanken und Gefühlen“ operationalisieren, eben deshalb als

68 Kolleg 1819, Jonas-Nachschrift, KGA II/4, 198.

69 Hermeneutik (1819), KGA II/4, 157. 
oberflächlich erscheinen. Ich möchte die Vermutung äußern, dass die emphatischen Übereinstimmungstopoi der frühromantischen Hermeneutik - in der optimistischen wie in der melancholischen Variante - einen stillschweigenden Themenwechsel gegenüber den Vorläuferprojekten der Aufklärungshermeneutik anzeigen. Auch wenn Schleiermacher die Hermeneutik allgemein als „die Kunst, die Rede eines anderen, vornehmlich die schriftliche, richtig zu verstehen“ ${ }^{70}$ bestimmt, ist der eigentliche Fluchtpunkt seines Unternehmens nicht das Textverstehen, sondern das notorisch schwer greifbare Personenverstehen. Das vornehmliche Interesse Schleiermachers gilt dem individuellen Ausdruckscharakter einer jeden menschlichen Äußerung. Wenn aber das zu Verstehende nicht mehr primär der Bedeutungsgehalt eines sprachlichen Gebildes ist, sondern ein menschliches Individuum in der ganzen Komplexität seiner biographischen Prägungen, Erfahrungen und psychischen Dispositionen, dann stoßen Verfahren der Auslegung schon deshalb an ihre Grenzen, weil der Verstehensgegenstand kein sprachförmiges Gebilde ist. Auf diesen kategorialen Unterschied zielt auch Gadamers Diktum „Sein, das verstanden werden kann, ist Sprache“, das Gadamer als Bemerkung über die „Grundverfassung von allem, auf das sich überhaupt Verstehen richten kann“, verstanden wissen will.. ${ }^{71}$

In eine ähnliche Richtung zielt Schillers Sinnspruch „Spricht die Seele, so spricht, ach! schon die Seele nicht mehr“. Die Seele als solche kann sich nicht aussprechen, weil sie nicht selbst sprachförmige Gestalt hat. Es ist nur eine Teilklasse der psychischen Zustände, die repräsentierenden Charakter hat und ein Format annehmen kann, das einer am Paradigma des Textverstehens orientierten Auslegung fähig ist. Für Davidson sind es die Inhalte propositionaler Einstellungen, die zum Gegenstand der Interpretation werden. Auch Schleiermacher bezieht seine Identitätsthese auf das Verhältnis von Sprechen und Denken, wobei er die Vorstellung, dass ein Gedanke nachträglich in ein sprachliches Format übersetzt werden müsste, als irrig zurückweist.

Bei näherer Betrachtung unterscheiden sich die Ambitionen von Schleiermachers Hermeneutikprojekt und von Davidsons Interpretationstheorie gravierend. ${ }^{72}$ Für Schleiermacher ist eigentlicher Fluchtpunkt und Ziel aller Verstehensbemühungen die Individualität des Sprechers, die er im Sinne von Goethes „individuum est ineffabile“ als unausschöpflich ansieht. In Davidsons ausgenüchtertem Projekt gibt es kein solches emphatisches Individualitätskonzept. Da

70 Schleiermacher, Hermeneutik und Kritik, Mit einem Anhang sprachphilosophischer Texte Schleiermachers, hg. von Manfred Frank, Frankfurt am Main 1977, 75.

71 Gadamer, Wahrheit und Methode, a.a.O. (Anm. 31), 478.

72 Diese Unterschiede sind mir durch Diskussionen mit Magnus Schlette klarer geworden, dem ich dafür zu Dank verpflichtet bin. 
sprachliche Bedeutung für Davidson - wie für Wittgenstein - ein wesentlich soziales und damit öffentliches Phänomen ist, kann nichts zur Bedeutung einer Äußerung gehören, was nicht im Prinzip aufgrund öffentlicher Belege verstehbar wäre. ${ }^{73}$ Spricht man statt vom Bedeuten vom Meinen, so ergibt sich nichts Neues, da wir nach Davidson mit unseren Worten nichts meinen können, wovon wir nicht beabsichtigen können, dass es verstanden wird.

Ob es sich bei der Fokussierung der romantischen Hermeneutik auf das Personenverstehen um einen Themenwechsel handelt oder nicht, hängt davon ab, ob man Hermeneutik allgemein als Kunst(lehre) des Verstehens oder als die des Textverstehens auffasst. Die letztere Auffassung dürfte die gebräuchlichere sein, aber der Begriff des Verstehens als solcher ist zu weit und zu schillernd, als dass Gadamers Diktum als eine analytische Wahrheit gelten könnte. Sein, das verstanden werden kann, ist nicht eo ipso ein sprachliches Gebilde. Wir sprechen zwanglos vom Verstehen von Personen, Handlungen, Entscheidungen, Gesten, Gesichtsausdrücken, Emotionen, historischen Entwicklungen und einigem anderem mehr.

Meiner Gegenüberstellung von Textverstehen und Personenverstehen wird man mit Blick auf Schleiermacher entgegenhalten, dass Textverstehen und Personenverstehen in der „psychologischen Interpretation“ notwendig ineinander übergehen und dass es Schleiermacher darum zu tun ist, die Individualität einer Person durch ihre sprachlichen Äußerungen zu verstehen. Nach dem Standardverständnis der psychologischen Interpretation ist die Vermittlungsrichtung zwischen Text- und Personenverstehen freilich die umgekehrte: Nach Schleiermacher ist ,jede Rede immer nur zu verstehen aus dem ganzen Leben, dem sie angehört“, nämlich „als Lebensmoment des Redenden in der Bedingtheit aller seiner Lebensmomente“. ${ }^{74}$ Es ist etwas anderes, biographisches und anderweitiges Wissen über eine Person zur Interpretation ihrer Äußerungen heranzuziehen, als die Person als solche zum Gegenstand von Verstehensbemühungen zu machen. Es ist das zweite Unternehmen, das die Aufgaben der Hermeneutik als Kunst der Auslegung sprachlicher Gebilde zumindest aus Gadamers Sicht überdehnt.

Wie genau das Sprachverstehen und das Personenverstehen sich zueinander verhalten, muss hier offen bleiben. Die Auffassung Humboldts jedenfalls, dass jedes Verstehen einer Äußerung aufgrund der unaufhebbaren wechselseitigen Intransparenz der Sprecher zugleich ein Missverstehen sei, schließt Textverstehen und Personenverstehen in bedenklicher Weise miteinander kurz. Es gehört nicht

73 „Die semantischen Merkmale der Sprache sind öffentliche Merkmale. Was niemand der Gesamtheit der Belege entnehmen kann, weil es aufgrund der Natur der Sache ausgeschlossen ist, das kann auch nicht mit zur Bedeutung gehören.“ Donald Davidson, „Die Unerforschlichkeit der Bezugnahme“ (1979), in: ders, Wahrheit und Interpretation, a.a.O. (Anm. 2), 321 - 340, hier 332. 74 Schleiermacher, Hermeneutik und Kritik, a.a.O. (Anm. 70), 78. 
zu den Aufgaben der philosophischen, literarischen oder theologischen Hermeneutik, die Melancholie ob der Unmöglichkeit der vollkommenen Übereinstimmung in Gedanken und Gefühlen aus der Welt zu schaffen. Dies gilt umso mehr, als das Kongruenzideal mit einem plausiblen Individuationsprinzip für Personen in Konflikt liegt. Vielleicht schwebt den Melancholikern in Wirklichkeit so etwas wie Platons Idee einer Verschmelzung mit der verlorenen anderen Hälfte vor. Diese unio mystica herbeizuführen liegt jenseits der Möglichkeiten und Aufgaben einer Kunst der Auslegung sprachlicher Äußerungen. 